\title{
MOBILIDADE URBANA E GÊNERO: PISTAS SOBRE A CIDADE HETEROGÊNEA
}

\author{
URBAN MOBILITY AND GENDER: CLUES ABOUT THE HETEROGENEOUS CITY
}

\begin{abstract}
RESUMO
Homens e mulheres circulam na cidade de maneira distinta em função das desigualdades de gênero histórica e socialmente produzidas. A cidade torna-se palco para uma heterogeneidade de experiências que, por sua vez, vão reproduzir a lógica de dominação masculina e as desigualdades estruturantes do sistema. Com o objetivo de analisar os fatores determinantes dessas diferenças, realizou-se uma revisão integrativa da produção científica dos últimos dez anos, utilizando os descritores "gênero" e "mobilidade urbana", recorrendo aos artigos disponíveis no Portal de Periódicos CAPES, Scielo e Biblioteca Virtual em Saúde Brasil. Foram selecionados 35 artigos para análise. Os resultados mostram que os estudos de gênero e mobilidade partilham pautas focalizadas em reivindicações sociais e apontam para a emergência de novas abordagens norteadas pelas epistemologias feministas e de abordagem interseccional, que fortalecem a ideia de mobilidade enquanto dispositivo, produto de inter-relações entre as estruturas espaciais, sociais e subjetivas, funcionando como uma poderosa tecnologia de governo e regulação dos corpos na cidade.
\end{abstract}

Palavras-chave: Gênero. Mobilidade Urbana. Desigualdades. Mulheres.

\section{ABSTRACT}

Men and women circulate in the city differently due to historical and socially produced gender inequalities. The city becomes the stage for heterogeneity of experiences that, in turn, will reproduce the logic of male domination and the structural inequalities of the system. To analyze the determinants of these differences an integrative review of the scientific production of the last ten years was carried out, using the descriptors "gender" and "urban mobility", with the articles available in the CAPES, Scielo, and Library Journal Portal Virtual Health in Brazil platforms. A total of 35 articles were selected for analysis. The results show that gender and mobility studies share guidelines focused on social demands and point to the emergence of new approaches guided by feminist epistemologies and an intersectional approach, which strengthen the idea of mobility as a device, a product of interrelationships between spatial, social,

Anderson de Andrade Silva

Doutorando em Psicologia pela Universidade Federal do Rio Grande do Norte. E-mail: andersonandrade17@gmail.com

Candida Maria Bezerra Dantas

Doutora em Psicologia - Professora Adjunta do Departamento de Psicologia da Universidade Federal do Rio Grande do Norte. E-mail: candida.dantas@gmail.com

Magda Diniz Bezerra Dimenstein

Doutora em Psicologia - Professora visitante da Universidade Federal do Ceará. Professora Titular vinculada ao Programa de Pós-Graduação em Psicologia da UFRN. E-mail: mgdimenstein@gmail.com 
and subjective structures, functioning as a powerful technology of government and regulation of bodies in the city.

Keywords: Gender. Urban mobility. Inequalities. Women.

\section{Introdução}

A análise das políticas e processos de mobilidade urbana através da perspectiva de gênero vem tomando corpo desde a década de 1970, em paralelo aos avanços no campo das lutas e teorias feministas. O interesse em compreender as diferenças e os padrões de circulação na cidade emerge como objeto teórico e metodológico resultante de reivindicações por uma cidade que possibilite a circulação equitativa para todas as pessoas, em particular, das mulheres no espaço público. Não à toa, em 2020, as desigualdades de gênero nas cidades tornam-se alvo prioritário de debate e investimento por parte de instituições internacionais preocupadas com os seus impactos sociais e econômicos, bem como com a busca de políticas mais equânimes que integrem o gênero no planejamento e governança das cidades, pensadas como lugar de cidadania. É o caso do documento produzido em parceria pelo Banco Interamericano de Desenvolvimento (BID), pelo Banco de Desenvolvimento da América Latina (CAF) e pela ONU-Habitat.

Os estudos sobre gênero e mobilidade ancoram-se na constatação de que as cidades não são pensadas a partir de uma ótica que toma o gênero como categoria fundamental de análise. Tomando Scott (1990) como referência, gênero é "uma forma primeira de significar as relações de poder" (SCOTT, 1990, p.21), "um elemento constitutivo de relações sociais baseado nas diferenças percebidas entre os sexos" (SCOTT, 1990, p.21), que articula as assimetrias de poder. A partir desse marcador social, essa discussão considera que homens e mulheres circulam na cidade de maneira distinta em função do acesso desigual aos recursos de mobilidade (HANSON, 1995; RAJU, 2005) e que isso promove diferenças nas atividades, nos padrões de viagens e na forma de vivenciar a cidade (FAGNANI, 1983; CRANE, 2007; VASCONCELLOS, 2012). Contudo, e mais fundamental, é que partem do princípio de que a cidade não se configura como um espaço neutro, mas como elemento de ordenamento das experiências urbanas a partir da lógica de dominação masculina e das desigualdades estruturantes do sistema, sejam de gênero, raça ou classe social (LYRA, 2020). De acordo com essa perspectiva, as lógicas patriarcais atravessam o espaço urbano e criam dicotomias que servem de reforço para os papéis socialmente estereotipados quanto ao feminino e ao masculino (VILLAGRÁN, 2014). Assim, a mobilidade urbana revela as tensões presentes no uso do espaço (BARBOSA, 2016), e, desse modo, seu entendimento exige considerar as relações de poder existentes nesse cenário.

Com base nesse pressuposto, alguns estudos têm investido na problematização de gênero em relação à mobilidade urbana (KUNIEDA e GAUTHIER, 2007; HANSON, 
2010; HELENE e TAVARES, 2017; ROLNIK, 2011, ESCALENE, 2017; BUJALANCE e NARANJO, 2012). Indicam, dentre outros aspectos, que as desigualdades de gênero em relação à mobilidade urbana são expressas na forma de circular na cidade, no uso dos meios de transportes, nos lugares onde o acesso está ou não autorizado e nos sentidos produzidos acerca da circulação de homens e mulheres. Apontam que as mulheres possuem padrões específicos de viagens que diferem dos padrões masculinos (ROSENBLOOM, 2006; KUNIEDA e GAUTHIER, 2007; TOBÍO, 2014) e que o deslocamento feminino é mais complexo comparado ao masculino (TOBÍO, 2014). Revelam que os padrões de deslocamento masculino tendem a serem lineares com único destino, com uma nítida referência ao movimento casa-trabalho (TOBÍO, 2014), além de serem marcados por um maior acesso aos veículos particulares (KUNIEDA e GAUTHIER, 2007). Já as mulheres, utilizam mais o transporte público e o caminhar para se locomoverem pela cidade (HAMILTON, 2002; KUNIEDA e GAUTHIER, 2007; TOBÍO, 2014;SVAB, 2016), realizam viagens com múltiplos destinos e propósitos (KUNIEDA e GAUTHIER, 2007) e apresentam, frequentemente, trajetos de menor distância, permanecendo em um raio próximo ao domicílio (HANSON e PRATT, 1995).

A busca por compreender os diferentes usos das cidades, segundo Helene e Tavares (2017), já figura há mais de três décadas em estudos urbanos europeus, canadenses e estadunidenses, com destaque para o campo da Geografia com o debate entre espaço e gênero. No Brasil, ainda são escassas as pesquisas que utilizam a perspectiva de gênero para compreender o uso das cidades e sua constituição. Destacamos os estudos de Lyra (2020) sobre a espacialização do medo e as opressões vividas pelas mulheres no espaço público; de Rolnik (2011) voltado às relações de gênero no direito à moradia, explicitando que as violações de direitos das mulheres resultam em consequências específicas e distintas quando comparada aos homens. Neste caminho, destacam-se os estudos de Silvia Rubino (2017), frutos dos esforços do Instituto Brasileiro de Direito Urbanístico (IBDU), de Paula Santoro (2019) e Terezinha Gonzaga (2004) que colocam no centro do urbanismo as necessidades cotidianas das pessoas que habitam as cidades, trazendo o recorte de gênero como possibilidade de interpretação para o desenvolvimento e uso dos equipamentos urbanos.

No campo dos estudos de mobilidade, percebe-se atualmente uma crise epistemológica no urbanismo científico, que desmonta os modelos baseados em metodologias positivistas, reducionistas e "neutras", ou seja, uma tendência em afastar-se dos estudos clássicos de planejamento urbano historicamente marcado por abordagens totalizadoras, voltado para uma ideia de coletivo homogêneo (HARKOT, LEMOS e SANTORO, 2018). No bojo desse esforço de complexificar a análise das relações entre gênero e mobilidade urbana, as epistemologias feministas, que "reclamam o caráter político da produção de conhecimento e denunciam o androcentrismo, o colonialismo, o universalismo e o eurocentrismo sobre o qual a ciência moderna está assentada" (FRANÇA e PADILLA, 2013, p. 1), vêm agregar a 
concepção de uma cidade plural, diversificada e heterogênea, que molda os desiguais modos de habitar e circular na cidade por homens e mulheres.

No entrecruzamento de olhares, a perspectiva interseccional, que "trata especificamente da forma pela qual o racismo, o patriarcalismo, a opressão de classe e outros sistemas discriminatórios criam desigualdades básicas que estruturam as posições relativas de mulheres, raças, etnias, classes e outras" (CRENSHAW, 2002, p. 177), aporta contribuições fundamentais à discussão sobre mobilidade urbana e gênero, já que "as pessoas habitam e transitam por espaços de forma diferente segundo gênero, raça, classe, sexualidade, geração e segundo a intersecção entre essas dimensões" (MAYORGA e RUEDA, 2019, p. 9).

No intuito de contribuir com esse debate, o presente trabalho buscou identificar, através de um levantamento bibliográfico, as diferentes dimensões abordadas pelos estudos que se dedicam a analisar a relação mobilidade urbana e gênero.

\section{Mobilidade urbana: da invisibilidade à visibilidade das práticas e particularidades de gênero}

Os estudos sobre a mobilidade urbana consolidaram-se como um tipo específico de reflexão sobre a circulação das pessoas e dos veículos, em um campo de disputas por espaços, por usos e por segurança. Em resposta a esse quadro complexo, entidades especializadas passam a dedicar-se exclusivamente ao estudo dessa dimensão urbana, vital para a vida nas cidades, pois ultrapassa o deslocarse, acrescentando meios fundamentais para a subsistência, para a vida familiar/ comunitária e às relações de gênero (HANSON, 2010).

Através dos estudos sobre os transportes urbanos, a Mobilidade Urbana tem sido pensada em torno de três paradigmas (CERVERO, 2013). No primeiro paradigma, observa-se o foco nas políticas de crescimento do tráfego baseadas nos veículos e na ampliação e criação de rodovias. Os esforços estão direcionados para estudos de transportes de larga escala, modelos de previsão de tráfego e distribuição de viagens (LANE, 1971; THOMPSON, 1969). Buscava-se maximizar a capacidade de veículos individuais e, metodologicamente, priorizava-se a análise quantitativa das relações entre transporte e uso do solo (JONES, 2014). O adensamento urbano e os vários problemas decorrentes disso evidenciaram a impossibilidade de atender o uso desenfreado dos automóveis, em especial, nas áreas urbanas mais densas, exigindo uma mudança das políticas em curso (PORTUGAL et al., 2017).

O segundo paradigma, ao invés de sustentar a movimentação ilimitada de veículos, objetiva problematizar o movimento das pessoas. Essa segunda perspectiva dá maior atenção à eficiência no deslocamento de pessoas de seu ponto de origem ao destino, destacando os transportes públicos como estratégia principal de intervenção (JONES, 2014). Uma produção marcante nessa mudança de perspectiva foi a publicação Downs-Thompson paradox, produzida a partir de estudos urbanos em 
Londres e Paris, onde Mogridge (1990) afirma a promoção de uma mudança política na oferta de meios adicionais de transporte para melhorar o deslocamento urbano. A partir da década de 1970, passou-se a questionar se essa perspectiva oferecia uma compreensão das finalidades e opções de viagens. Os estudos costumavam focar nos percursos e não priorizavam os motivos envolvidos, a organização e o planejamento do espaço urbano não era considerado (PORTUGAL et al., 2017). Desse modo, abriu-se a possibilidade para outro tipo de compreensão que se consolida no terceiro paradigma, onde não basta apenas pensar na efetivação da mobilidade, mas no planejamento e na distribuição dos equipamentos urbanos na cidade como elementos decisivos. Portanto, as atividades das pessoas e os objetivos envolvidos em cada deslocamento, tornam-se as principais preocupações. O movimento - em seu sentido restrito - assumiu uma posição secundária (JONES, 1983). A requalificação da rua exige intervenções específicas, a exemplo da cidade de Portland em São Francisco e Seul na Coreia do Sul (ITDP, 2012), onde grandes estruturas viárias estão sendo demolidas para promover outras formas de uso da cidade (JONES, 2004).

Assim, no terceiro paradigma, a finalidade do deslocamento passa a ter maior importância que o deslocamento em si. Preocupações associadas à qualidade de vida, promoção social e estilos de vida sustentáveis tornam-se fundamentais. É nesse contexto que a interface entre gênero e mobilidade urbana passa a ser alvo de discussões, já que a intenção é atender aos requisitos voltados para as atividades das pessoas, trazendo à tona as especificidades dos grupos citadinos. Anteriormente, os estudos sobre a mobilidade urbana não só invisibilizava as mulheres, como também não oferecia nenhuma alternativa crítica para a superação desse cenário. É importante ressaltar ainda que essa divisão não expressa que um paradigma superou completamente o outro, contudo, percebe-se que é mais apropriado pensar em sua sobreposição (HEIGGE e JONES, 1978).

Percebe-se, pois, uma guinada na tendência dos estudos sobre mobilidade urbana, focada nos veículos e na ampliação de rodovias, e passam a se aproximar cada vez mais das pessoas. A escala da cidade mudou, o olhar se volta para as relações, para as atividades envolvidas em cada percurso, em uma cidade cada vez mais complexa. Nesse sentido, as contribuições da perspectiva de gênero aos estudos de mobilidade urbana foram decisivas, pois tratam de construções sociais que definem identidades tanto para as mulheres quanto para os homens e, desse modo, os papéis sociais a eles atribuídos.

As relações de gênero constroem-se no cotidiano e estão evidentemente relacionadas às práticas de mobilidade na cidade (PRATT e HANSON, 1994). Destaca-se que a mobilidade não está resumida a mera reprodução de estruturas sociais, ela também opera como produtora dessas diferenças (JIRÓN e SINGH, 2017), sendo eminentemente uma categoria política na medida em que expressa, autoriza, contesta ou produz relações de poder (CRESSWELL, 2010).

A expressão de gênero pode ser entendida enquanto performance, uma identidade que é construída por uma teia complexa de elementos e que não possui uma correspondência diretiva com o sexo biológico (BUTLER, 2019). Gênero não pode 
ser compreendido dentro de uma estrutura permanente, gênero é sempre um efeito, ou seja, uma identidade que está em relação, “(...) essa identidade é performativamente constituída, pelas próprias 'expressões' tidas como seus resultados” (BUTLER, 2019, p. 56). Desse modo, trata-se de uma identidade que é construída das referências sociais disponíveis e também para além delas. A noção de gênero proposta por Butler (2019) aponta que sua constituição se dá através dos atos, gestos, representações, e isso nos oferece uma ferramenta importante para compreender a mobilidade urbana ao direcionarmos o olhar para as pessoas e seu circular pela cidade através da ideia de performatividade, potencializando uma leitura distante de qualquer ideia homogeneizante do que é ser mulher.

Butler (2019) constrói uma genealogia crítica das categorias de gênero, onde recusa uma leitura da categoria através de interpretações essencializadas, contudo, propõe uma investigação a partir de causas múltiplas, ou seja, as identidades “(...) são efeitos de instituições, práticas e discursos cujos pontos de origem são múltiplos e difusos" (BUTLER, 2019, p. 9). A partir de uma leitura foucaultiana, Butler (2019) nos apresenta a necessidade de compreender o conceito de gênero através de um conjunto interdisciplinar e pós-disciplinar entre os discursos. Sexo e gênero são inscritos no corpo, construídos e inscritos socialmente. Essa estrutura promove o entendimento de gênero como o efeito constituído de poderes particulares (MCLAREN, 2016). Os estudos de gênero de inspiração foucaultiana, a exemplo de Judith Butler (2019), Jana Sawicki (1991) e Susan Hekman (2014) aportam recursos para afirmar que gênero é continente de uma subjetividade que é corporificada e constituída historicamente através das relações sociais. Esse entendimento possibilita, como argumenta Mclaren (2016), um método que ajuda a questionar as relações de poder que são hegemônicas e materializadas.

Sobre a relação gênero e mobilidade, uma contribuição importante foi trazida pelo pensamento da geógrafa feminista Susan Hanson (2010). A autora propõe duas grandes tendências nos estudos que articulam gênero e mobilidade urbana. Considera que elas partem de diferentes entendimentos sobre gênero, mobilidade e contexto, apresentando metodologias distintas e focos de pesquisa igualmente diferentes. A primeira tendência parte da ideia de que a mobilidade e a imobilidade estão presentes na criação, reforço e mudança dos significados e práticas de gênero. Segundo Hanson (2010), os autores dessa tendência "emphasized gender to the relative neglect of mobility, in that authors have paid a great deal of attention to illuminating, in considerable detail, the ways in which the gendered dimensions of power, agency, identity and subjectivity relate to mobility" (HANSON, 2010, p. 9). Em outras palavras, enfatizaram o gênero, compreendendo que a mobilidade molda o gênero, porém, negligenciaram a mobilidade em seus estudos. Esses trabalhos indicaram um entendimento de como a mobilidade ou imobilidade estão implicadas na criação, manutenção e transformação dos significados e práticas de gênero (HANSON, 2010). A segunda tendência situa suas reflexões na questão de como o gênero molda a mobilidade (HANSON, 2010, p. 11), e está ancorada, em grande medida, nas pesquisas quantitativas que evidenciam uma visão simplificada 
de gênero, resumida ao sexo biológico e investem na metrificação detalhada da mobilidade. Dados secundários, jornadas para o trabalho e origem - destino são objetos privilegiados desses estudos, onde a categoria gênero se apresenta como uma variável binária (masculino e feminino), com o objetivo de confrontar as diferenças entre distâncias, tempo percorrido, modo de viagem, entre homens e mulheres (HANSON, 2010).

Cada uma dessas duas tendências expõe suas lacunas ao direcionar o foco para uma das categorias em detrimento da outra, apresentando-se sempre como concepções parciais. Hanson (2010) conclui que seria necessário novos esforços para a produção de uma terceira direção que pudesse sintetizar as duas vertentes, de uma maneira contextualizada, associando estudos quantitativos e qualitativos com o objetivo de construir leituras ampliadas que pudessem abarcar a complexidade desse fenômeno. Ademais, é imprescindível discutir de quais mulheres estamos tratando. Torna-se evidente que a categoria mulher não comporta as infinitas possibilidades. Segundo Singh (2018) a primeira intenção dos estudos de gênero sobre mobilidade é abandonar a ideia que existe um sujeito universal que se move. A categoria gênero como pressuposto de uma única identidade torna-se produtora de um discurso colonizador (BUTLER, 2019). Para Butler (2019, p. 21), é impossível dissociar gênero dos marcadores sociais como raça, classe, etnia, regionalidades, sexualidades. Ele é produzido nessas interseções. A mobilidade se estabelece, dessa maneira, enquanto experiência urbana e deve ser estudada através dessas intersecções, pois, como diz Gouveia (2016, p.10), "uma coisa é constatar a presença das mulheres na cidade, outra completamente distinta é pensar a produção desse espaço tendo como preocupação política e analítica, a estrutura e a dinâmica das relações das desigualdades entre mulheres e homens". Assim, almeja-se contribuir na produção de melhores condições urbanas, e através do ponto de vista feminino, produzir um diagnóstico eficiente da cidade e de seus recursos, pois pensar uma cidade justa para as mulheres é pensar numa cidade justa para todos (BUJALANCE e NARANJO, 2012).

\section{Procedimentos metodológicos}

O presente estudo consiste de uma revisão integrativa da literatura, articulando as categorias gênero e mobilidade. A composição do material foi realizada mediante busca de artigos indexados nas bases eletrônicas de dados: Portal de Periódicos CAPES, Scielo e Biblioteca Virtual em Saúde Brasil, com os seguintes descritores "gênero" AND “mobilidade urbana”. Foram incluídos, para fins de análise neste estudo, os artigos publicados nos últimos dez anos, ou seja, entre maio de 2010 e maio de 2020. Foi estabelecido como critério de inclusão o acesso on-line ao artigo completo de forma gratuita e revisado por pares. Foram definidos como critérios de exclusão: artigos científicos duplicados e outros materiais que não fossem artigos científicos. Após remoção dos artigos que atenderam aos critérios de exclusão foi 
realizada a leitura integral dos resumos dos materiais classificados. Por fim foram excluídos os artigos que não apresentavam articulação com o foco do estudo, a saber: problematizar a mobilidade como uma tecnologia de governo dos corpos na cidade. A coleta do material ocorreu entre maio e junho de 2020. Inicialmente foram identificados 75 artigos. Após aplicar os critérios de inclusão e exclusão elegemos 35 artigos para o banco de dados final. A síntese da estratégia de busca é melhor detalhada no quadro a seguir:

Figura 1 - Representação gráfica do processo de seleção do banco de dados.

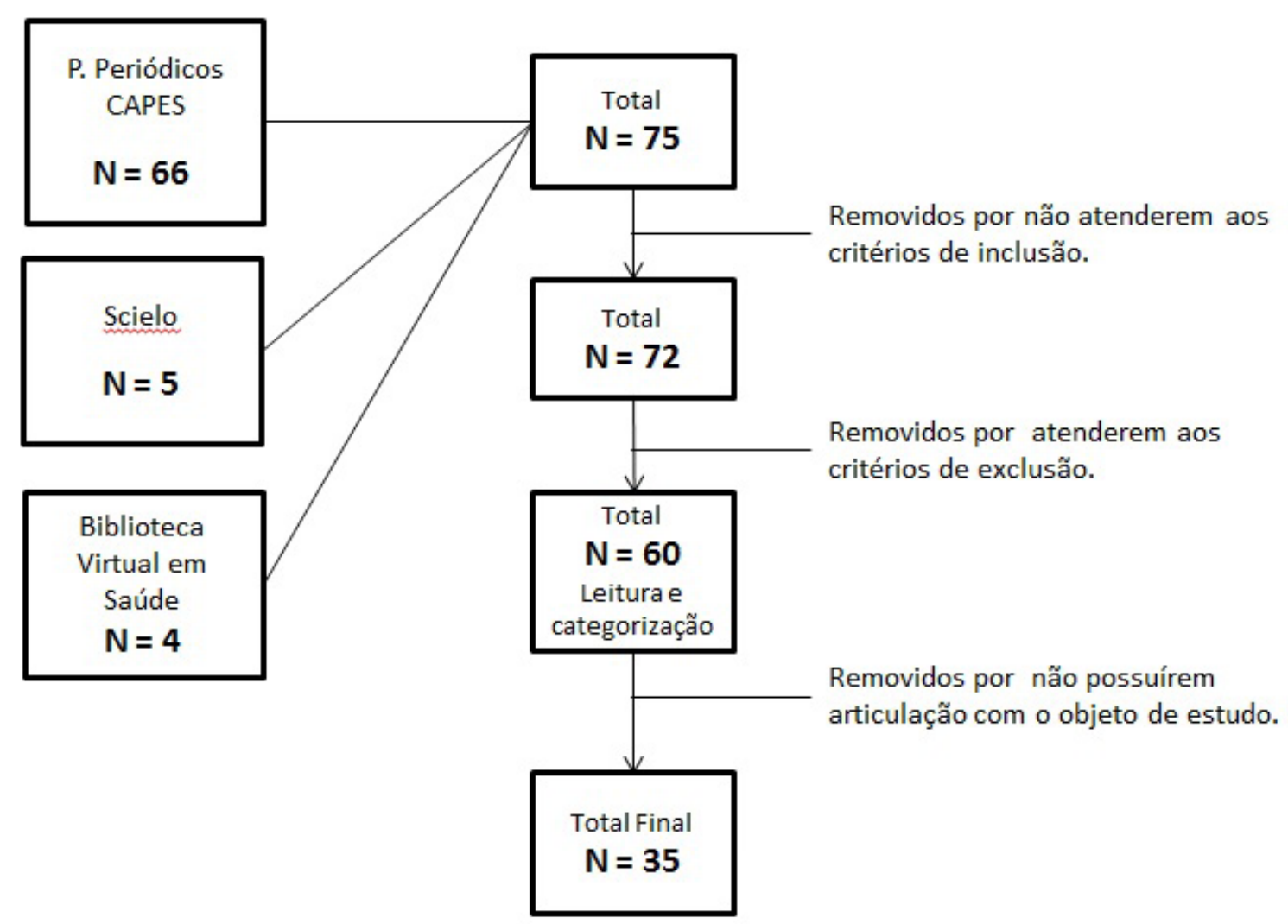

Todos os artigos selecionados foram inseridos em uma matriz para organização e análise. A matriz era composta pelos itens: ano, área do conhecimento, sexo dos autores, local onde o estudo foi realizado, estudo teórico ou empírico, desenho metodológico, grupo/objeto estudado, principais conceitos que o texto maneja, referencial teórico, conclusão geral do artigo e articulação do artigo com o objetivo do estudo. 


\section{Resultados e Discussão}

O panorama geral dos artigos selecionados mostra duas tendências. A primeira trata de 26 artigos que abordam os conceitos de gênero e mobilidade urbana, porém não estabelece uma relação de interdependência entre eles. A segunda tendência explora as categorias sob uma perspectiva relacional e articulada entre gênero e mobilidade, evidenciando como o gênero molda a mobilidade e vice-versa, totalizando 9 artigos.

Na primeira tendência identificamos expressiva produção no ano de 2018 com 9 artigos, seguido do ano de 2016 com 4 trabalhos, com destaque para uma publicação em 2020 de Rios et al (2020). Observamos uma proporção equilibrada em relação ao sexo dos autores de 50\% do sexo masculino e 50\% do sexo feminino. Sobre a natureza do estudo, há a mesma proporção entre os teóricos e os empíricos. Quanto ao desenho metodológico, há prevalência de estudos qualitativos (80,8\%), seguido por $15,4 \%$ de estudos quantitativos e 3,8\% de estudos mistos. Destaca-se o artigo de Gohn (2014a), compreendendo a América Latina e o trabalho europeu de Zilhão (2013) situado na cidade do Porto em Portugal. Os demais estão focados na realidade brasileira com expressiva produção de trabalhos no eixo sul e sudeste do país.

No segundo bloco de estudos que estabelecem uma relação direta entre gênero e mobilidade, identificamos o primeiro material a partir de 2015 (PIANÇO, 2015). São de natureza teórica e/ou empírica (PIANÇO, 2015; SORJ, 2016; PAVARINO, 2016; SINGH 2018; HARKOT, LEMOS e SANTORO, 2018). No que se refere ao desenho metodológico um pouco mais da metade são estudos qualitativos e 44,5\% de estudos quantitativos e nenhum estudo de caráter misto. Estão concentrados na realidade brasileira, com exceção de Singh (2018) e Páramo e Burbano (2019), que contemplam a América Latina e Colômbia, respectivamente. Quanto aos autores, destaca-se a produção das mulheres, já que $23,5 \%$ são homens e $76,5 \%$ são mulheres. Isso sugere que as experiências cotidianas das autoras na cidade é um fator que contribui para o entendimento de uma relação direta entre gênero e mobilidade urbana e para a necessidade de produção do conhecimento a partir desse pressuposto. Após a leitura na íntegra, os artigos foram organizados em quatro blocos temáticos: equidade de gênero, sustentabilidade, justiça social e reivindicações de direito à cidade, os quais serão descritos a seguir.

\section{Equidade de gênero}

O escopo teórico do material investigado oferece alguns achados interessantes. Zilhão (2013) argumenta que a compreensão da centralidade do ordenamento urbano exige um investimento teórico e metodológico multidisciplinar, face à sua complexidade, os variados significados adquiridos em função dos marcadores sociais 
e seus efeitos singulares para homens e mulheres. Guimarães (2016) argumenta, através de uma análise interseccional, que a mobilidade também opera na segregação racial quando relaciona classe, condição periférica e mercado imobiliário, ou seja, que a experiência negra e periférica é impactada pelas longas distâncias e precariedade nos transportes. Os estudos indicam que, enquanto ferramenta teórico-metodológica, a interseccionalidade desvela "os processos de interação entre relações de poder e categorias como classe, gênero e raça em contextos individuais, práticas coletivas e arranjos culturais/institucionais." (RODRIGUES, 2013, p.1).

Os desafios da mobilidade metropolitana contemporânea revelam a necessidade de ampliar o entendimento da mesma sob a perspectiva de gênero. Percursos seguros e eficientes para todas e todos refletem um desenvolvimento urbano satisfatório (DE GÓIS, 2018). No âmbito da internacionalização das cidades, ou seja, de uma cidade global, inserida nos roteiros de turismo, de produção e consumo de bens, esses níveis satisfatórios de mobilidade são esperados (NARA et al, 2016; JESUS, 2017). O estudo de Hino e Cunha (2017) evidenciam a interferência da perspectiva de gênero na percepção relativa à importância de tecnologias para alguns serviços de mobilidade urbana, especialmente para o transporte público inteligente.

Pesquisas sobre espaços definidos oferecem uma aproximação ainda maior às peculiaridades que a categoria de gênero imprime na mobilidade. $O$ contexto territorial é mais um elemento importante. Páramo e Burbano (2019) investem na leitura socioespacial do ato de caminhar em Bogotá e afirmam que para as mulheres (no contexto estudado) caminhar não é apenas arriscado, mas também limitado pelo desenho espacial e pelas barreiras simbólicas que condicionam o acesso ao espaço público. A geografia do gênero garante que não é seguro para as mulheres circularem em espaços públicos da cidade, por conta das distintas violências e violações. Concluem que, em Bogotá, as mulheres se sentem constantemente assediadas ao circularem pela cidade (PÁRAMO e BURBANO, 2019).

Seguindo a linha de estudos contextualizados Harkot, Lemos e Santoro (2018) apresentam uma agenda de pesquisa sobre mobilidade ativa (bicicleta) e gênero em São Paulo, modalidade que se revela problemática diante das desigualdades de gênero, pois as mulheres são, geralmente, excluídas do sistema de mobilidade urbana. Isso produz uma desproporcionalidade enorme entre homens e mulheres que usam a bicicleta como meio de locomoção. A situação é tão hostil que a mera existência de mulheres nas ciclovias pode ser um possível indicador de qualidade urbana (SOUSA e PENHA-SANCHES, 2019). Em outras palavras, esse grupo de estudos sugere que para existir uma condição equânime na mobilidade urbana entre homens e mulheres torna-se indispensável uma articulação interseccional contextualizada no território, visando à superação das desigualdades de classe, raça/etnia, dentre outras. 


\section{Sustentabilidade}

Existe um aparato legal que orienta a mobilidade urbana (BRELAZ e ALVES, 2013; GRANDO e DE BONA, 2018) e, dentro dessas normativas, as questões de gênero são citadas como uma dimensão importante de implementação, principalmente quando está associada à sustentabilidade (TONELLA, 2013) ou incluída nas políticas públicas (PINTO e VILLAMIZAR-DUARTE, 2018). Chama a atenção o fato dos autores supracitados tratarem a categoria gênero como uma alternativa teórico-metodológica em prol de melhorias na mobilidade urbana, mesmo não deixando claro como isso pode ser operacionalizado.

Alinhado aos princípios ecológicos e de sustentabilidade, a mobilidade urbana, para alguns autores, vai se aproximando das teorias de gênero (BARROS, 2015; OLIVEIRA, 2018), chegando à aposta em uma mobilidade "feminina", e, nesse sentido, pode haver uma generalização arriscada. A ideia de uma mobilidade feminina é proveniente da hipótese de que as mulheres fazem um uso da cidade com menor impacto ambiental, pois utilizam mais o transporte coletivo e a caminhada para se locomoverem pela cidade. Porém esse fato não representa, necessariamente, escolhas, mas pode estar associado às limitações e constrangimentos que as mulheres enfrentam cotidianamente nos seus deslocamentos.

Dessa forma, Hanson (2010) alerta que ao estabelecer que a mobilidade das mulheres é mais "sustentável", é preciso conhecer os motivos que produzem as menores distâncias percorridas e o menor uso de automóveis, como também é preciso superar a noção homogeneizante no que se refere às mulheres, já que muitos estudos abordam um coletivo indiferenciado de mulheres que a própria literatura feminista já explicou não existir (RAGO, 1998; BUTLER, 2019).

\section{Justiça social}

Tomando gênero e mobilidade como operadores de um conjunto complexo de determinações, Pianço (2015) explora a noção de Trabalho Decente com jovens adultos para destacar as desigualdades existentes entre homens e mulheres. Para o autor, Trabalho Decente é o

Trabalho produtivo com remuneração justa, segurança no local de trabalho e proteção social para o trabalhador e sua família; melhores perspectivas para o desenvolvimento pessoal e social; liberdade para que manifestem suas preocupações, organizemse e participem na tomada de decisões que afetam suas vidas; e igualdade de oportunidades e de tratamento para as mulheres e homens. (PICANÇO, 2015, p. 569 apud OIT, 2007a). 
Ele, é, portanto, um indicador de justiça social. Nesse sentido, o fato das mulheres enfrentarem maior dificuldade na mobilidade urbana, resulta em maior tempo no deslocamento de casa para o trabalho e repercute em piores condições de trabalho. Essa vulnerabilidade se amplia quando as mulheres passam a sobrepor trabalho e atividades conjugais: “(...) as mulheres mais afastadas dos papéis tradicionais de gênero são aquelas com mais acesso ao trabalho decente" (PIANÇO, 2015, p. 582). Assim, as condições sociais normativas de gênero não só repercutem na mobilidade, mas também produzem efeitos nas condições de trabalho, e isso deveria interferir na construção de uma agenda de políticas públicas para o trabalho, em especial para mulheres jovens.

Sorj (2016) aponta que a mobilidade urbana é um dos elementos principais em relação à qualidade do trabalho e que as mulheres são penalizadas nessa situação, especialmente as que moram em periferias e que precisam fazer longos deslocamentos até os centros urbanos ou bairros mais abastados. Essa condição fragiliza a justiça social (PIANÇO, 2015; SORJ, 2016). A partir de uma leitura crítica do relatório da ONU Mulher, intitulado Progress of the World's Women 2015-2016, Sorj (2016) esclarece que a ausência de debate sobre as migrações internacionais e a mobilidade urbana, produz ainda maior vulnerabilidade para as mulheres, já que se trata de um debate sobre desigualdades de classe e de gênero.

Os relatórios/materiais que por vezes servem de marco regulatório nos ajudam a pensar propostas de enfrentamento às demandas apresentadas. Pavarino Filho (2016) produz um trabalho semelhante ao de Sorj (2016), porém utiliza as declarações sobre a segurança rodoviária de dois importantes centros urbanos (Brasília e Moscou). Segundo o autor é preciso "integrar plenamente a perspectiva de gênero em todos os processos de tomada de decisão e de implementação de políticas relativas à mobilidade e à segurança no trânsito, em especial nas vias, ambientes de circulação e no transporte público" (PAVARINO FILHO, 2016, p. 3655), como sinaliza a Segunda Conferência Global de Alto Nível sobre Segurança no Trânsito: Tempo de Resultados, Brasília, novembro de 2015 na $\mathrm{OP} 18$. Enfim, os estudos analisados explicitam que as relações de gênero e mobilidade se estabelecem num nível amplo e complexo e estão associadas à justiça social, com impacto na igualdade de direitos das experiências coletivas, pois limitam e precarizam o acesso das mulheres a diversos dispositivos urbanos.

\section{Reivindicações de direito à cidade}

A mobilidade urbana está envolta num campo de disputas e, por vezes, conflitam com as pautas feministas. Os movimentos emergentes no cenário urbano, estruturados por ações reivindicatórias de bens e equipamentos, apesar de ocuparem espaços semelhantes, não necessariamente lutam pelas mesmas coisas. Acesso à mobilidade urbana e equidade de gênero são objetivos que se aproximam, mas 
nas produções teóricas de parte dos autores analisados não se sobrepõem (GOHN, 2014a; GOHN 2014b; SCHERER-WARREN, 2014; SHIMIZU et al, 2015; DE SOUSA TEODÓSIO et al, 2016; LÜCHMANN, SCHAEFER e NICOLETTI, 2017; GOHN, 2018; FRÚGOLI, 2018; BARROS, 2018; TATAGIBA e GALVÃO, 2019). Tais trabalhos compartilham da ideia de gênero e mobilidade urbana como reivindicações de movimentos sociais. Os estudos posteriores a junho de 2013, momento que produziu uma sequência de protestos contrários ao aumento da tarifa de transporte coletivo na cidade de São Paulo, carregam essa marca. Disparada pela frase "não é só pelos vinte centavos", ficou evidente que as questões de mobilidade urbana podem emergir como uma pauta mais ampla e importante nas reivindicações sociais e isso terá reflexo nas pesquisas da área.

Cavalcanti et al (2019) vão além da lógica denunciativa de muitos estudos e apresentam, dentro da mesma adversidade já descrita, um movimento de resistência tomando o ciclismo como lugar de transformação das relações de gênero na cidade. Nesse aspecto, a mobilidade molda o gênero. O estudo etnográfico acompanhou um grupo de ciclistas liderado por mulheres e analisou a construção do empoderamento das participantes, mas também as dificuldades de trânsito na cidade, que não apresenta infraestrutura adequada, indicando, mais uma vez, que os elementos urbanos são imperativos e relacionados à perspectiva de gênero no direito à cidade.

\section{Considerações finais}

A produção científica analisada sobre gênero e mobilidade urbana pôde ser categorizada através dos blocos temáticos: reivindicações de direito à cidade, equidade de gênero, sustentabilidade e justiça social, sugerindo que essas dimensões estão na pauta do dia e do entendimento contemporâneo sobre a discussão. Este artigo objetivou mapear as contribuições dos estudos sobre a temática, sobre as desigualdades existentes entre homens e mulheres. Esses estudos, norteados pelas epistemologias feministas e abordagem interseccional, auxiliam no entendimento de que a mobilidade urbana pode ser considerada uma poderosa tecnologia de governo e regulação dos corpos na cidade. Segundo Manderscheid, "patterns of movement and mobility practices emerge in specific, historically - and personally - shaped social and spatial relations" (MANDERSCHEID, 2014, p.4). Assim, tal como Lemos et al. (2016), consideramos que é imprescindível pensar nas estratégias biopolíticas na cidade contemporânea centradas na capitalização dos espaços e dos corpos, tomando a mobilidade tanto como um dispositivo de sujeição, quanto de insurreição das subjetividades e das experiências urbanas femininas. 


\section{Referências}

BARBOSA, J. L. O Significado da mobilidade na construção democrática da cidade. In: BALBIN, R. KRAUSE, C. LINKE, C. C. (orgs.). Cidade e Movimento: mobilidades e interações no desenvolvimento urbano. Brasília: Ipea: ITDP, 2016, p. 43-56.

BARROS, Antonio Teixeira de. Juventude e poder político local: a percepção e o discurso de jovens sobre as eleições municipais de 2016. Sociedade e Estado, Brasília, vol. 33, n. 3, 2018, p. 849-886.

BARROS, Antonio Teixeira de. Política partidária e meio ambiente: a adesão dos partidos políticos brasileiros à agenda verde. Opinião Pública, Campinas, vol. 21, n. 3, 2015, p. 693-733.

BID; CAF; ONU Habitat. As desigualdades de gênero nas cidades. 2020, p. 10-102. Disponível em: <https://publications.iadb.org/publications/portuguese/document/ As_desigualdades_de_genero_nas_cidades.pdf> Acesso em: 27 nov. 2020.

BRELÀZ, Gabriela de; ALVES, Mário Aquino. The process of institutionalization of participation in the City House of Sao Paulo: an analysis of budget public hearings (1990 to 2010). Revista de Administração Pública, Rio de Janeiro, vol. 47, n. 4, 2013, p. $803-826$.

BUJALANCE, Susana Garcia; NARANJO, María Lourdes Royo. La perspectiva de género en el urbanismo. Una aproximación conceptual adaptada. In: Investigación y género, inseparables en el presente y en el futuro: IV Congreso Universitario Nacional Investigación y Género, [libro de actas]. Facultad de Ciencias del Trabajo de la Universidad de Sevilla, 21 y 22 de junio de 2012. (Coord.) Isabel Vázquez Bermúdez; (Com. cient.) Consuelo Flecha García et al. Sevilla: Unidad para la Igualdad, Universidad de Sevilla. Universidad de Sevilla, 2012, p. 609-626.

BUTLER, Judith. Problemas de gênero: feminismo e subversão da identidade. Rio de Janeiro: Civilização Brasileira, 2019.

CAVALCANTI, Tássia de Souza et al. "Eu sou Barbie e sou bruta": o empoderamento no ciclismo. Revista Estudos Feministas, Florianópolis, vol. 27, n. 2, 2019, p. 1-14.

CERVERO, Robert. Linking urban transport and land use in developing countries. Journal of transport and land use, Berkeley, vol. 6, n. 1, 2013, p. 7-24.

CRANE, Randall. Is there a quiet revolution in women's travel? Revisiting the gender gap in commuting. Journal of the American planning association, Berkeley, vol. 73, n. 3, 2007, p. 298-316. 
CRESSWELL, Tim. Towards a politics of mobility. Environment and Planning D: Society and Space, London, vol. 28, 2010, p. 17-31.

DE GÓIS, Marcos Paulo Ferreira. Mobilidade noturna: estudo sobre os circuitos urbanos noturnos na cidade do Rio de Janeiro. Universitas Humanística, Rio de Janeiro, vol. 85, n. 85, 2018, p. 263-291.

DE SOUSA TEODÓSIO, Armindo dos Santos et al. Sociedade Civil e Governo na Gestão de Cidades: a incidência do Movimento Nossa BH nas políticas públicas. Future Studies Research Journal: Trends and Strategies, São Paulo, vol. 8, n. 2, 2016, p. 27-53.

ESCALENE, Sara Ortiz. Urbanismo desde la perspectiva de género. Buenas prácticas con perspectiva de derechos humanos - Col-lectiu Punt 6, Barcelona, 2017, p. 5-9. Disponível em: <http://www.corteidh.or.cr/tablas/r3610o.pdf> Acesso em: 10 maio 2020.

FAGNANI, J. Women's commuting patterns in the Paris region. Tijdschrift voor Economische en Sociale Geografie, Paris, vol. 24, 1983, p. 12-24.

FRANÇA, Thais; PADILLA, Beatriz. Epistemologias feministas e mobilidade científica: contribuições para o debate. Configurações. Revista de sociologia, Braga, n. 12, 2013, p. $47-60$.

FREHSE, Fraya. Em busca do tempo nas ruas e praças de São Paulo. Ponto Urbe. Revista do núcleo de antropologia urbana da USP, São Paulo, n. 18, 2016, p. 1-17.

FRÚGOLI JR, Heitor. Ativismos urbanos em São Paulo. Caderno CRH, Salvador, vol. 31, n. 82, 2018, p. 75-86.

GOHN, Maria da Glória Marcondes. Gestão social e compromisso social solidário/ Social management and social commitment in solidarity. Revista Metropolitana de Sustentabilidade, São Paulo vol. 4, n. 2, 2014b, p. 82-95.

GOHN, Maria da Glória. Jovens na política na atualidade-uma nova cultura de participação. Caderno CRH, Salvador, vol. 31, n. 82, 2018, p. 117-133.

GOHN, Maria da Glória Marcondes. Pluralidade da representação na América Latina. Sociedade e Estado, Brasília, vol. 29, n. 1, 2014a, p. 73-90.

GONZAGA, Terezinha de Oliveira. A cidade e a arquitetura também mulher: planejamento urbano, projetos arquitetônicos e gênero. São Paulo: Annablume, 2004. 
GOUVEIA, Taciana. Mulheres: sujeitos ocultos das/nas cidades?. 2016, p. 1-10. Disponível em: <https://pt.scribd.com/document/235282224/80-Genero-e-ReformaUrbana-g-Mulheres-Sujeitos-Ocultos-Dasnas-Cidades>. Acesso em: 27 nov. 2020.

GRANDO, Fabiane; DE BONA, Celito. O aspecto jurídico da sustentabilidadeinstrumentos normativos regulamentadores das licitações sustentáveis. Revista de Direito e Sustentabilidade, Porto Alegre, vol. 4, n. 2, 2018, p. 20-39.

GUIMARÃES, Antonio Sérgio Alfredo. National formations of class and race. Tempo Social, São Paulo vol. 28, n. 2, 2016, p. 161-182.

HAMILTON, Kerry. Gender and transport in developed countries. In: Background paper for the workshop Gender Perspectives for Earth Summit. Berlin, 2002, p. 1-42.

HANSON, Susan. Gender and mobility: new approaches for informing sustainability. Gender, Place $\mathcal{E}$ Culture, vol. 17, n. 1, 2010, p. 5-23. Disponível em: <https://www. tandfonline.com/doi/pdf/10.108o/o9663690903498225?needAccess=true> Acesso em: 8 maio 2020.

HANSON, Susan; PRATT, Geraldine. Gender, work and space. New York: Routledge, 1995 .

HARKOT, Marina Kohler; LEMOS, Letícia Lindenberg; SANTORO, Paula Freire. Como as mulheres de São Paulo usam a cidade? Uma análise a partir da mobilidade por bicicleta. Dearq, Bogotá n. 23, 2018, p. 56-63.

HEGGIE, Ian G.; JONES, Peter M. Defining domains for models of travel demand. Transportation, Oxford vol. 7, n. 2, 1978, p. 119-125.

HEKMAN, S. J. The feminine subject. Cambridge: Polity Press, 2014.

HELENE, D.; TAVARES, R. T. Gênero e estudos urbanos, uma conciliação necessária. ArchDaily, 2017. Disponível em: <https://www.archdaily.com.br/br/881263/genero-eestudos-urbanos-uma-conciliacao-necessaria> Acesso em: 15 maio 2020.

HINO, Marcia R. Martelozo Cassita; DA CUNHA, Maria Alexandra VC. Mobilidade urbana: estudo da importância de serviços baseados em tecnologia para os gêneros. Revista Electronica de Sistemas de Informação, Campo Largo, vol. 16, n. 1, 2017, p. 1-17.

ITDP and EMBARQ, The Life and Death of Urban Highways. Washington: ITDF, 2012. 
JESUS, Diego Santos Vieira de. The Art of Encounter: Paradiplomacy and Internationalization of Creative Cities. Revista de Sociologia e Política, Curitiba, vol. 25, n. 61, 2017 p. 51-76.

JIRÓN, Paola; SINGH, Dhan Zunino. Dossier. Movilidad urbana y género: experiencias latinoamericanas. Revista Transporte y Territorio, Buenos Aires, n. 16, 2017, p. 1-8.

JONES, Peter. The evolution of urban mobility: The interplay of academic and policy perspectives. IATSS Research, London vol. 38, n. 1, 2014, p. 7-13.

KUNIEDA, Mika; GAUTHIER, Aimee. Sustainable Transport: A sourcebook for policy-makers in developing cities. Module 7a: gender and urban transport: smart and affordable, 2007, p. i-40. Disponível em: <https://itdpdotorg.wpengine.com/wpcontent/uploads/2014/o7/7aGenderUTSept3oo.pdf> Acesso em: 8 maio 2020.

LANE, Robert; POWELL, Timothy J.; SMITH, Paul Prestwood. Analytical transport planning. London: Bloomsbury Academic, 1971.

LEMOS, Flávia Cristina Silveira et al. Cidades, corpos medicalizados e o biocapital: o mercado da saúde. Fractal: Revista de Psicologia, Rio de Janeiro, vol. 28, n. 2, 2016, p. 187-194.

LÜCHMANN, Lígia Helena Hahn; SCHAEFER, Márcia Inês; NICOLETTI, André Selayaran. Associativismo e repertórios de ação político-institucional. Opinião Pública, Campinas, vol. 23, n. 2, 2017, p. 361-396.

LYRA, Júlia de Freitas Correia. Sujeitas públicas, narrativas anônimas: Espacializando o medoea (im)permanência da mulher noâmbito urbano em Maceió/AL - Brasil, Forum Sociológico [Online], n. 36, 2020. Disponível em: <http://journals.openedition.org/ sociologico/8991> Acesso em: 27 nov. 2020.

MANDERSCHEID, Katharina. Criticising the solitary mobile subject: Researching relational mobilities and reflecting on mobile methods. Mobilities, London, vol. 9, n. 2, 2014, p. 188-219.

MAYORGA, Claudia; RUEDA, Lupicinio Íñiguez. Gênero, feminismo e cidades. URBS: Revista de estudios urbanos y ciencias sociales, Almería vol. 9, n. 1, 2019, p. 9-15.

MCLAREN, Margaret A. Foucault Feminismo e Subjetividade. São Paulo: Intermeios, 2016.

MOGRIDGE, Martin JH. Travel in towns: jam yesterday, jam today and jam tomorrow? London: Macmillan, 1990. 
NARA, Elpidio Oscar Benitez et al. Análise de mobilidade do sistema viário do campus sede da Universidade de Santa Cruz do Sul. Exacta, São Paulo vol. 14, n. 2, 2016, p. 221233.

NOBRE, Lauro. Desafios e soluções para a mobilidade urbana. Caderno FGV projetos - Cidades Inteligentes e Mobilidade Urbana, Rio de Janeiro, n. 24, 2014, p. 120-129.

OLIVEIRA, Meilyng Leone. Desenvolvimento sustentável e os municípios: uma análise sob a perspectiva dos objetivos do desenvolvimento sustentável e da lei no 13.493/17 (PIV-PRODUTO INTERNO VERDE). Revista de Direito e Sustentabilidade, Salvador, vol. 4, n. 1, 2018, p. 59-76.

Organização das Nações Unidas (ONU). Census Bureau. Worldometers, World Statistics Updated in Real Time, 2016. Disponível em: <http://www.worldometers. info/world-population/brazil-population/> Acesso em: 10 maio 2020.

PÁRAMO, Pablo e BURBANO, Andrea. La caminabilidad en Bogotá: propósitos y condiciones socioespaciales que facilitan y limitan esta experiencia. Revista de arquitectura, Bogotá, vol. 21, n. 2, jul/dez. 2019, p. 12-21.

PAVARINO FILHO, Roberto Victor. The Moscow and Brasilia Declarations on road safety-a parallel between two moments in health. Ciencia $\mathcal{E}$ saude coletiva, Brasília, vol. 21, 2016, p. 3649-366o.

PICANÇO, Felícia Silva. Juventude e trabalho decente no Brasil-uma proposta de mensuração. Caderno CRH, Salvador, vol. 28, n. 75, 2015, p. 569-59o.

PINTO, Ana Marcela Ardila; VILLAMIZAR-DUARTE, Natalia. Ciudad (anía) en movimiento: construcción social de instrumentos de políticas de movilidad en Bogotá y Belo Horizonte 1995-2015. Universitas Humanística, Bogotá, vol. 85, n. 85, 2018, p. 20-57.

PORTUGAL, Licinio da Silva et al. Transporte, mobilidade e desenvolvimento urbano. Rio de Janeiro: Elsevier, 2017.

PRATT, Geraldine; HANSON, Susan. Geography and the construction of difference. Gender, Place and Culture: a journal of feminist geography, vol. 1, n. 1, 1994, p. 5-29. Disponívelem:<https://www.tandfonline.com/doi/abs/10.108o/o9663699408721198> Acesso em: 5 maio 2020.

RAGO, Margareth. Epistemologia feminista, gênero e história. Masculino, feminino, plural. Florianópolis: Ed. Mulheres, 1998. 
RAJU, Saraswati. Gender and empowerment: creating 'thus far and no further' supportive structures, a case from India. In: NELSON, Lise \& SEAGER, Joni (orgs.). A companion to feminist geography. Malden: Blackwell Publishing, 2005, p. 194-207.

RIOS, Polianna Alves Andrade et al. Factors associated with traffic accidents among drivers: findings from a population-based study. Ciência E Saúde Coletiva, vol. 25, 2020, p. 943-955. Disponível em: <https://www.scielosp.org/pdf/csc/2020. v25n3/943-955/pt> Acesso em: 10 maio 2020.

RODRIGUES, Cristiano. Atualidade do conceito de interseccionalidade para a pesquisa e prática feminista no Brasil. In: Anais do Seminário Internacional Fazendo Gênero. Florianópolis: UFSC/ UDESC, 2013, p. 1-12.

ROLNIK, Raquel et al. Como fazer valer o direito das mulheres à moradia. Relatoria especial da ONU para o direito à moradia. São Paulo: LabCidade | FAUUSP, 2011.

ROSENBLOOM, Sandra. Understanding women's and men's travel patterns. In: ROSENBLOOM, Sandra; FERGUSON, SusanA.; HANDY, Susan L. et al (orgs.). Research on women's issues in transportation: Report of a conference. Washington: TRB, 2004, p. 7-28.

RUBINO, Silvana. Lugar de mulher: arquitetura e design moderno, gênero e domesticidade. Tese (Livre Docência em História da Arquitetura e do Urbanismo) Instituto de Filosofia e Ciências Humanas. Campinas: Universidade de Campinas, 2017.

SANTORO, Paula Freire. Gênero e planejamento territorial: uma aproximação. In Anais do XVI Encontro Nacional de Estudos Populacionais. Caxambu: ABEP, 2019, p. 1-16.

SAWICKI, Jana. Disciplining Foucault: Feminism, power, and the body. New York: Routledge, 1991.

SCHERER-WARREN, Ilse. Dos movimentos sociais às manifestações de rua: o ativismo brasileiro no século XXI. Política \& Sociedade, Florianópolis, vol. 13, n. 28, 2014, p. 1334 .

SCOTT, Joan. Gênero: uma categoria útil deanálise histórica. Educação \& realidade, vol. 20, n. 2, 1995, p. 71-99. Disponível em: <https://www.seer.ufrgs.br/educacaoerealidade/ article/viewFile/71721/40667>. Acesso em: 15 maio 2020. 
SHIMIZU, Helena Eri et al. The structure of social representations on health and illness among members of social movements. Ciencia $\mathcal{E}$ saude coletiva, Rio de Janeiro, vol. 20, n. 9, 2015, p. 2899-2910.

SINGH, Z. Dhan. Cities, practices, and representations in motion: notes for a cultural analysis of mobility as an urban experience. Tempo Social, São Paulo, vol. 30, n. 2, 2018, p. 35-54.

SORJ, Bila. Igualdade de gênero e políticas macroeconômicas. Revista Estudos Feministas, Florianópolis, vol. 24, n. 2, 2016, p. 617-620.

SOUSA, Isabel-Cristina Nunes de; PENHA-SANCHES, Suely da. Fatores influentes na escolha de rota dos ciclistas. EURE (Santiago), Santiago, vol. 45, n. 134, 2019, p. 31-52.

SVAB, Haydée. Evolução dos padrões de deslocamento na região metropolitana de São Paulo: a necessidade de uma análise de gênero. Tese de Doutorado. São Paulo: Universidade de São Paulo, 2016.

TATAGIBA, Luciana; GALVÃO, Andreia. Os protestos no Brasil em tempos de crise (2011-2016). Opinião Pública, Campinas, vol. 25, n. 1, 2019, p. 63-96.

THOMSON, John Michael. Motorways in London. London: Gerald Duckworth \& Co Ltd, 1969.

TOBÍO, Constanza. Estructura urbana, movilidad y género en la ciudad moderna. Boletín CF+ S, Madrid, n. 13, 2014, p. 1-9.

TONELLA, Celene. Políticas Urbanas no Brasil: marcos legais, sujeitos e instituições. Sociedade e Estado, Brasília, vol. 28, n. 1, 2013, p. 29-52.

VASCONCELLOS, E. Transporte Urbano, Espaço e Equidade: Análise das Políticas Públicas. 1a. ed. São Paulo: Annablume, 2001.

VILLAGRÁN, Paula Soto. Patriarcado y orden urbano. Nuevas y viejas formas de dominación de género en la ciudad. Revista venezolana de estudios de la mujer, Caracas, vol. 19, n. 42, 2014, p. 199-214.

ZILHÃO, Adriano. Uma proposta multidisciplinar para o entendimento da centralidade urbana como facto social total. Sociologia, Revista da Faculdade de Letras da Universidade do Porto, Porto, vol. 25, 2013, p. 55-77.

Recebido em 30/10/2020.

Aceito em 23/11/2020. 